\title{
ARTICLE \\ Moderators for depressed mood and systemic and transcriptional inflammatory responses: a randomized controlled trial of endotoxin
}

\author{
Michael R. Irwin (D ${ }^{1,2}$, Steve Cole ${ }^{1}$, Richard Olmstead ${ }^{1}$, Elizabeth C. Breen ${ }^{1}$, Joshua Jin Cho (iD) ${ }^{1}$ Mona Moieni ${ }^{1,2}$ and \\ Naomi I. Eisenberger ${ }^{2}$
}

\begin{abstract}
Activation of the innate immune system is thought to contribute to depression. Multiple social and behavioral factors are also known to instigate depression. Whether these socio-behavioral factors interact with inflammatory stimuli to alter proinflammatory responses and depressed mood is not known. In 115 healthy adults, social, emotional, and behavioral factors were assessed at baseline. A single infusion of endotoxin (Escherichia coli; $0.8 \mathrm{ng} / \mathrm{kg}$ of body weight) or placebo ( $0.9 \%$ saline) was administered with hourly assessment of depressed mood and proinflammatory cytokines (interleukin-6 (IL-6); tumor necrosis factor-a (TNF)). Inflammatory gene expression was examined at $30 \mathrm{~min}$ after infusion, prior to increase of inflammatory cytokines. As compared to placebo, endotoxin-induced increases of depressed mood were moderated by baseline levels of perceived stress, trait sensitivity to social disconnection, and severity of symptoms of anxiety and depression (all Ps $<0.05$ ) but not early life stress, social status, social support, neuroticism, or sleep disturbance. Anxiety symptoms remained significant in multivariable analyses $(P<0.01)$. None of these socio-behavioral factors were related to increases in proinflammatory cytokines. Transcriptome profiling analyses indicated that perceived stress, sensitivity to social disconnection, and depressive symptoms were associated with increased activation of pro-inflammatory transcription control pathways (i.e., activator protein-1, nuclear factor-kB) in response to endotoxin (all Ps $<0.05$ ). These results indicate that an array of socio-behavioral factors, which are associated with depression risk, modify vulnerability to inflammation-induced depressed mood. Together, these observations may be used to help target therapeutic interventions to mitigate occurrence of the inflammatory biotype of depression.
\end{abstract}

Neuropsychopharmacology (2019) 44:635-641; https://doi.org/10.1038/s41386-018-0259-6

\section{INTRODUCTION}

Depression, among the most common of all psychiatric disorders [1], is a biologically heterogeneous disorder. In addition, social factors (i.e., social rejection) [2] and behavioral symptoms (i.e., anxiety, sleep disturbance) [2-5] are proximal, as well as independent, predictors of depression onset. To prospectively identify those at risk and to inform development of targeted strategies for the prevention and treatment of depression, it is critical to understand how these conceptually distinct sociobehavioral factors interact with biological mechanisms to induce depressive symptoms.

One biological phenotype of depression is thought to stem from excessive activation of the innate immune system [6]. Some depressed patients show elevated levels of systemic inflammatory markers [7], along with increased expression of inflammationrelated genes in monocytes or peripheral blood mononuclear cells $[8,9]$. Moreover, among the substantial minority (i.e., about $30 \%$ ) of patients with elevated levels of inflammation [10], treatment response varies along with inflammation [10-13].

Inflammation may have a causal role in inducing the inflammatory biotype of depression. In those with an inflammatory disorder, depression is three times more prevalent [2], and inflammation prospectively predicts incident depression [14]. Moreover, experimental induction of inflammation using endotoxin [15-20], pharmacologic doses of interferon (IFN)-a [21, 22], or typhoid vaccination [23] can induce depressive symptoms in non-depressed adults and alter neural activity in depressionrelevant brain systems $[16,17,23]$. However, there is striking heterogeneity in inflammatory and depression responses to inflammatory stimuli. Indeed, female sex $[19,20]$ and sleep disturbance [24], two risk factors of depression, heighten vulnerability to depressed mood in response to inflammatory challenge.

Socio-behavioral factors, implicated in enhancing inflammation [25], may also contribute to the inflammatory biotype of depression [2]. The social signal transduction hypothesis of depression suggests that individuals' perceptions of adversity (e.g., perceived stress) are shaped by several influences, including social-environmental conditions during childhood and adulthood (e.g., early life stress, perceived social status, social support), as well as emotional traits (e.g., neuroticism, sensitivity to social disconnection) $[2,19,20]$. In response to perceived stress, these socio-emotional factors amplify symptoms of anxiety, as well as depressive symptoms and sleep disturbance, which in turn

\footnotetext{
${ }^{1}$ Cousins Center for Psychoneuroimmunology, Jane and Terry Semel Institute for Neuroscience and Human Behavior, Department of Psychiatry and Biobehavioral Sciences, David Geffen School of Medicine, University of California, Los Angeles, CA, USA and ${ }^{2}$ Department of Psychology, University of California, Los Angeles, CA, USA Correspondence: Michael R. Irwin (mirwin1@ucla.edu)
}

Received: 3 August 2018 Revised: 4 October 2018 Accepted: 24 October 2018 Published online: 3 November 2018 
activate social signal transduction pathways that upregulate proinflammatory mediators (i.e., interleukin-6 (IL-6), tumor necrosis factor-a (TNF)) [2]. Given evidence that anxiety, as well as anxiety disorders, temporally precede the onset of depressive disorders and that anxiety and depression perhaps arise from a similar personality trait or biological process [26, 27], as further evidenced by shared genetic risk variance [28], anxiety symptoms may have a unique role in amplifying inflammation-induced depressed mood. Proinflammatory transcriptional changes in circulating leukocytes are also reported to be associated independently with many of these factors, including perceived stress, early life stress, low perceived social status, sensitivity to social disconnection, and sleep disturbance $[2,29]$; such skewing of the leukocyte basal transcriptome toward a proinflammatory state may increase the likelihood of depressive responses [29-31].

This study examined the effect of experimental challenge with low-dose endotoxin vs. placebo on depressed mood and inflammatory responses in a large sample of healthy adults and tested whether baseline levels of socio-emotional variables (i.e., perceived stress, early life stress, perceived social status, social support, neuroticism, trait sensitivity to social disconnection $[19,20]$ ) and behavioral symptoms (i.e., state of anxiety or depressive symptoms, sleep disturbance) moderated the magnitude of depressed mood responses, as well as the magnitude of response of proinflammatory cytokines (i.e., IL-6, TNF). For those factors that moderated depressed mood responses, we also examined moderating effects on two key proinflammatory transcription control pathways known to mediate leukocyte responses to endotoxin activation of the lipopolysaccharide receptor-activator protein (AP)-1 and nuclear factor (NF)-кB. Moreover, the state of anxiety, as a proximal predictor of depression, was hypothesized to have a salient role in moderating greater increases in depressed mood in response to endotoxininduced inflammatory exposure as compared to placebo.

\section{METHODS}

Participants

One hundred and fifteen healthy participants (mean age $24.2 \pm 6.6$ years; 69 females and 46 males) completed a randomized study of endotoxin vs. placebo administration (Fig. 1S) [19, 20]. Inclusion and exclusion criteria were previously described $[19,20]$. All subjects provided written consent; procedures were approved by the UCLA Human Subjects Protection Committee.

Procedures

This randomized, double-blind, placebo-controlled study was conducted between March 2011 and August 2013. (NCT01671150) Partial results previously reported are: varying depressed mood responses due to sex $[19,20]$ or pre-existing sleep disturbance [24], varying inflammatory response due to sensitivity to social disconnection $[19,20]$, endotoxin effects on positive and negative social feedback [32], and activation of the ventral striatum in relation to social reward [33]. No prior study has comprehensively and simultaneously examined the moderating roles of multiple socio-behavioral factors on depressed mood and proinflammatory responses to an experimental inflammatory challenge.

Each participant was randomly assigned (RO) by computergenerated 1:1 allocation algorithm to receive either low-dose endotoxin $(0.8 \mathrm{ng} / \mathrm{kg}$ of body weight, E. coli group O:113 BB-IND 12948 to MRI) as provided by the National Institutes of Health Clinical Center [34] or placebo (same volume of $0.9 \%$ saline) as an intravenous bolus. To verify inflammatory activation, circulating levels of IL- 6 and TNF were measured at baseline (T0) and then every hour post-administration over $6 \mathrm{~h}$ (T1-T6); endotoxin administration led to over ten-fold increases of IL-6 and TNF levels [19, 20], consistent with findings by others [35], and comparable to endotoxin as currently formulated (CCRE Reference Endotoxin PDS Lot: 139926, Manufacturer Lot: 94332B1). This dose of endotoxin mimics increases in inflammation as found in inflammatory disorders, infections, and psychological stress [36-38]. Blood was collected for gene expression analyses at two timepoints (T0 and 30 min later, T0.5), because peak RNA response precedes peak protein levels $[19,20]$. Depressed mood and physical sickness symptoms were assessed from T0 to T6 [19, 20].

\section{Primary outcome}

Change in depressed mood from T0 to T2 was the primary outcome as assessed by the depression subscale of the short-form Profile of Mood States (POMS) [39]. The T2 timepoint was selected because endotoxin induces peak increases in depressed mood at $2 \mathrm{~h}$ after exposure. Temporal profile of POMS depressed mood from T0 to T6, controlling for T0 levels, was secondarily examined as an outcome $[19,20]$

\section{Secondary outcome}

Change in plasma levels of IL-6 and TNF across the temporal profile from T0 to T6 was a secondary outcome $[19,20]$, as quantified by high-sensitivity bead-based multiplex (Luminex) immunoassays (Performance High Sensitivity Human Cytokine, R\&D Systems, Minneapolis, MN, USA) [19, 20].

A related secondary outcome was change from T0 to T0.5 in proinflammatory signaling activity in peripheral blood mononuclear cells (PBMC), assessed using bioinformatic quantification of pro-inflammatory transcription factor activity derived from linear model analysis of (log2-transformed) gene expression profiling data. Transcriptome profiling analyses were conducted solely to provide point estimates of differential gene expression and to serve as input into the bioinformatics analysis of transcription factor activity. Analyses of transcription factor activity were conducted on a "protected testing" basis (i.e., only for sociobehavioral variables that emerged as statistically significant moderators of endotoxin effects on depressed mood. Genomewide transcriptional profiling was conducted on isolated PBMC using Ambion TotalPrep cRNA targets hybridized to Illumina HT-12 v4 bead arrays, as performed in the UCLA Neuroscience Genomics Core Laboratory following the manufacturer's standard protocol $[19,20]$. Of the 115 participants, 111 provided blood samples at both T0 and T0.5; all paired samples yielded sufficient RNA for analysis, were assayed in a single batch, and provided valid results according to standard data quality metrics (e.g., median probe fluorescence intensity $>100$ units).

\section{Moderators of outcome}

Demographic and clinical variables. Background characteristics including age, sex, and educational level were obtained, along with assessment of body mass index (BMI); these variables were entered as covariates because each is associated with depression risk, inflammation, or both [2].

Socio-emotional variables. Socio-emotional measures were obtained at T0; this set of variables was selected because each has been found to be a proximal, and independent, risk factor for inflammatory activity or depression [2] including perceived stress (i.e., Perceived Stress Scale) [40]; early life stress (i.e., Risky Families Questionnaire) [41]; perceived social status (i.e., Scale of Subjective Social Status) [42]; social support (i.e., short form Social Support Questionnaire) [43]; neuroticism (i.e., Eysenck Personality Inventory) [44]; and trait sensitivity to social disconnection [19, 20] (i.e., a composite of the Brief Fear of Evaluation Scale [45], Mehrabian Sensitivity to Rejection Scale; [46], UCLA Loneliness Scale [47], and anxious attachment subscale of the Experiences in Close Relationships-Revised questionnaire [48] (see refs. $[19,20]$ ). 
Behavioral measures. Symptoms of anxiety and depressive symptoms were also assessed at T0 (i.e., Beck Depression and Beck Anxiety Inventories) [49] to evaluate the state of symptom severity during the preceding week ("the past week, including today"). The Pittsburgh Sleep Quality Index was used to assess perceived sleep disturbance [50].

\section{Sample size}

For changes in depressed mood (i.e., primary outcome), endotoxin administration has a moderate effect (0.52) [15-20], and 50 per treatment group provides a statistical power of $90 \%(a=.05)$.

Statistical analysis

Given that each of the selected socio-behavioral factors has been found to contribute uniquely to depression [2], separate univariate analyses of covariance (ANCOVA), controlling for covariates (i.e., age, sex, years of education, and $\mathrm{BMI}$ ), were first used to examine separate moderator main effects and interaction with endotoxin vs. placebo condition on change in depressed mood from T0 to T2; values were log transformed owing to positive skew. Secondary analyses using ANCOVA $[19,20]$ examined each moderator main effect and interaction with endotoxin vs. placebo condition on the temporal profile of POMS depressed mood from T0 to T6, covarying for T0 baseline levels of depressed mood and covariates. Analyses were adjusted for multiple comparisons by Simes correction. These moderators have known independent effects on depression and inflammation [2]. However, because several moderators share overlapping variance (i.e, correlations between neuroticism and depressive symptoms $(r=0.62, P<$ $0.001)$, depressive and anxiety symptoms $(r=0.59, P<0.001)$, and perceived stress and trait sensitivity to social disconnection $(r=0.56, P<0.001))$, a multivariate analysis of variance was used to assess the relative importance of moderators.

For secondary outcome of IL-6 and TNF, moderators were tested on the temporal profile of cytokines levels from T0 to T6, covarying for respective T0 baseline levels and above covariates; values were log transformed for normality. IBM-SPSS version 24 was used.

For bioinformatic analyses of transcription factor activity, raw gene expression data were quantile-normalized [51] and log2transformed for general linear model analyses quantifying the magnitude of within-subject change in transcript abundance from T0 to T0.5 (i.e., a pre-post difference score) as a function of a main effect of condition, main effect of moderator (expressed as a $z$ score), and condition $\times$ moderator term, with the above covariates. Hence, this analysis examined response differences between endotoxin and placebo conditions at the two timepoints and whether socio-behavioral factors differentially influence responses, accounting for possible T0 differences in gene expression as well as the relation of condition and of moderator to T0. To identify transcription control pathways that might vary in parallel with differential responses of depressed mood to endotoxin as a function of the moderator, TELiS promoter-based bioinformatics analyses [52] were performed on all gene transcripts showing a point estimate of $\geq 1.2$-fold in the condition $\times$ moderator interaction term (i.e., magnitude of difference in the endotoxin vs. placebo effect over the range from -2 SD to +2 SD relative to mean value of the moderator) $[19,20]$. The 1.2-fold effect size threshold was pre-specified by prior research [53] to generate differentially expressed gene sets with a balance between specificity and statistical power for downstream analysis of transcription factor activity. These putatively associated genes were subject to TELiS promoter-based bioinformatic analysis to assess activity of NF-KB (using the TRANSFAC V\$NFKB_Q6 transcription factor-binding motif weight matrix) and AP-1 (V \$AP1_Q6). Although not hypothesized a priori, ancillary analyses tested for potential moderation of Type I IFN responses using the V\$ISRE_01 weight matrix. Transcription factor activity was assessed by the log-ratio of transcription factor-binding motifs in the promoter sequences of upregulated genes vs. downregulated genes (ensuring that all analyzed genes can potentially be expressed in PBMC and avoiding cell-type-specific bias in transcript expression patterns), with results averaged across 9 parametric combinations of 3 promoter sequence lengths $(-300$ bp upstream of the RefSeq gene transcription start site, $-600 \mathrm{bp}$, and -1000 to $+200 \mathrm{bp}$ ) and 3 stringencies for motif detection (Transfac mat_sim values $\geq 0.80,0.90$, and 0.95 ). Statistical inference for bioinformatics results was based on standard errors derived by bootstrapping of linear model residuals (200 cycles of resampled residual vectors, which controls for any potential correlation among residuals across genes) [54]. Low-level transcript-phenotype associations (Supplement, Differentially Expressed Genes) were estimated solely to provide point estimate inputs into high-level TELiS gene set analyses and were not tested for statistical reliability at the level of individual genes. Although changes in composition of circulating immune cells can occur with potent inflammatory stimuli that induce sympathetic activation, endotoxin $(0.8 \mathrm{ng} / \mathrm{kg})$ induces only modest increases in sympathetic activation as indexed by increases in heart rate, [35] with such increases found at 2-3 h, but not within the first hour (i.e., T0.5); transcript origin analyses was not performed in the absence of anticipated change in primary cell source of differentially expressed genes from T0 to T0.5.

\section{RESULTS}

Baseline sample characteristics

Figure 1S (Supplement) shows the flow of subjects [19, 20]. Table 1S (Supplement) provides the descriptive characteristics of the sample at baseline T0, with no group differences between the conditions in any of the socio-emotional or behavioral variables except for higher perceived social status and lower levels of sleep disturbance in the endotoxin group $(P s<0.05)$.

\section{Moderators of change of depressed mood in response to} endotoxin

Socio-emotional variables. Perceived stress and sensitivity to social disconnection interacted with endotoxin vs. placebo and predicted greater increases in depressed mood in the endotoxin condition ( $P_{\mathrm{s}}<0.05$; Table 1; Fig. 1). However, within the endotoxin condition itself, neither perceived stress nor sensitivity to social disconnection was related depressed mood. Neither early life stress, perceived social status, social support, nor neuroticism interacted with endotoxin vs. placebo to predict differences in change in depressed mood.

Behavioral symptoms. Severity of baseline levels of self-reported anxiety $(P<0.001)$ and depressive symptoms $(P \mathrm{~s}<0.05)$, but not sleep disturbance, interacted with endotoxin vs. placebo to predict greater increases in depressed mood in the endotoxin condition (Table 1; Fig. 2). Within the endotoxin condition itself, self-reported anxiety, but not depressive symptoms or sleep disturbance, predicted increases in depressed mood $(P=0.002)$.

Adjustment for multiple comparisons did not substantially alter results, in which moderator interactions remained for perceived stress $(P=0.03)$, sensitivity to social disconnection $(P=0.06)$, anxiety symptoms $(P=0.004)$, and depressive symptoms $(P=$ 0.02 ). Finally, given sex differences of depressed mood in response to endotoxin vs. placebo $[19,20]$, sensitivity analyses evaluated whether the moderator effects were sex specific; moderator results for perceived stress, sensitivity to social disconnection, and anxiety and depressive symptoms did not differ by sex (all PS > 0.2). Whereas female sex predicts greater depressed mood response to endotoxin, sex does not alter the influence of sociobehavioral moderators on depressed mood response.

For temporal profile of depressed mood from T0 to T6, similar 
Table 1. Baseline moderators of change in depressed mood from baseline to $\mathrm{T} 2$ in response to endotoxin vs. placebo condition, univariate results

\begin{tabular}{|c|c|c|c|c|}
\hline & Covariate $\left(B^{\mathrm{a}}\right)$ & $P$ & $\begin{array}{l}\text { Condition } \times \\
\text { covariate }\left(B^{\mathrm{b}}\right)\end{array}$ & $P$ \\
\hline \multicolumn{5}{|l|}{ Social-emotional } \\
\hline Perceived stress & 0.058 & 0.37 & 0.296 & $<0.01$ \\
\hline Early life stress & -0.044 & 0.34 & -0.081 & 0.24 \\
\hline $\begin{array}{l}\text { Perceived social } \\
\text { status }\end{array}$ & 0.019 & 0.48 & 0.007 & 0.85 \\
\hline Social support & 0.160 & 0.09 & 0.101 & 0.38 \\
\hline Neuroticism & -0.015 & 0.39 & -0.040 & 0.09 \\
\hline $\begin{array}{l}\text { Trait sensitivity } \\
\text { to social } \\
\text { isolation }\end{array}$ & 0.061 & 0.14 & 0.123 & 0.03 \\
\hline \multicolumn{5}{|l|}{ Behavioral symptoms } \\
\hline $\begin{array}{l}\text { Anxiety } \\
\text { symptoms }\end{array}$ & 0.015 & $<0.002$ & 0.038 & $<0.001$ \\
\hline $\begin{array}{l}\text { Depressive } \\
\text { symptoms }\end{array}$ & 0.015 & 0.15 & 0.039 & $<0.01$ \\
\hline $\begin{array}{l}\text { Sleep } \\
\text { disturbance }\end{array}$ & -0.003 & 0.91 & 0.040 & 0.20 \\
\hline
\end{tabular}

results were obtained with moderation by perceived stress, sensitivity to social disconnection, and baseline levels of anxiety and depressive symptoms (all $P \mathrm{~s}<0.05$; data not shown).

Relative contribution of socio-emotional and behavioral variables. In multivariate analyses, anxiety remained a significant moderator to predict increases in depressed mood in response to endotoxin $(P<0.01)$, considering simultaneous contribution of all other socio-behavioral factors (Table $2 \mathrm{~S}$, Supplement).

Moderators of inflammatory response to endotoxin

Secondary analyses examined whether moderators interacted with endotoxin to predict changes from T0 to T6 in IL-6 and TNF levels. Only sensitivity to social disconnection interacted with endotoxin vs. placebo and predicted greater increases of TNF in the endotoxin condition $(P<0.05)$, as previously reported $[19,20]$, although this effect was no longer significant after adjusting for multiple comparisons ( $P>0.1$; Table $3 \mathrm{~S})$.

Moderators of inflammatory transcriptional profiles to inflammatory challenge

To explore whether proinflammatory gene transcription activity might be moderated by the select set of socio-behavioral variables that were related to depressed mood responses to endotoxin (i.e, perceived stress, sensitivity to social disconnection, and symptoms of anxiety and depression), we analyzed genome-wide transcriptional profiles of PBMC sampled at T0 and T0.5 after endotoxin (or placebo). The observed patterns of differential gene expression were utilized to assess the magnitude of activation in two proinflammatory transcription control pathways: AP-1/Fos-Jun family of transcription factors and NF-KB/Rel family of transcription factors.

Analyses identified 17 gene transcripts that were upregulated $>1.2$-fold by endotoxin exposure (Supplement, Differentially Expressed Genes), including the pro-inflammatory genes, IL1B, IL8, TNF, PTGS2 (COX2), and CCL3; the dendritic cell activation marker CD83; the NF-KB-induced transcripts NFKBIZ and NFKBIA (IKB-alpha and -zeta); the AP-1 family member FOSB; and the
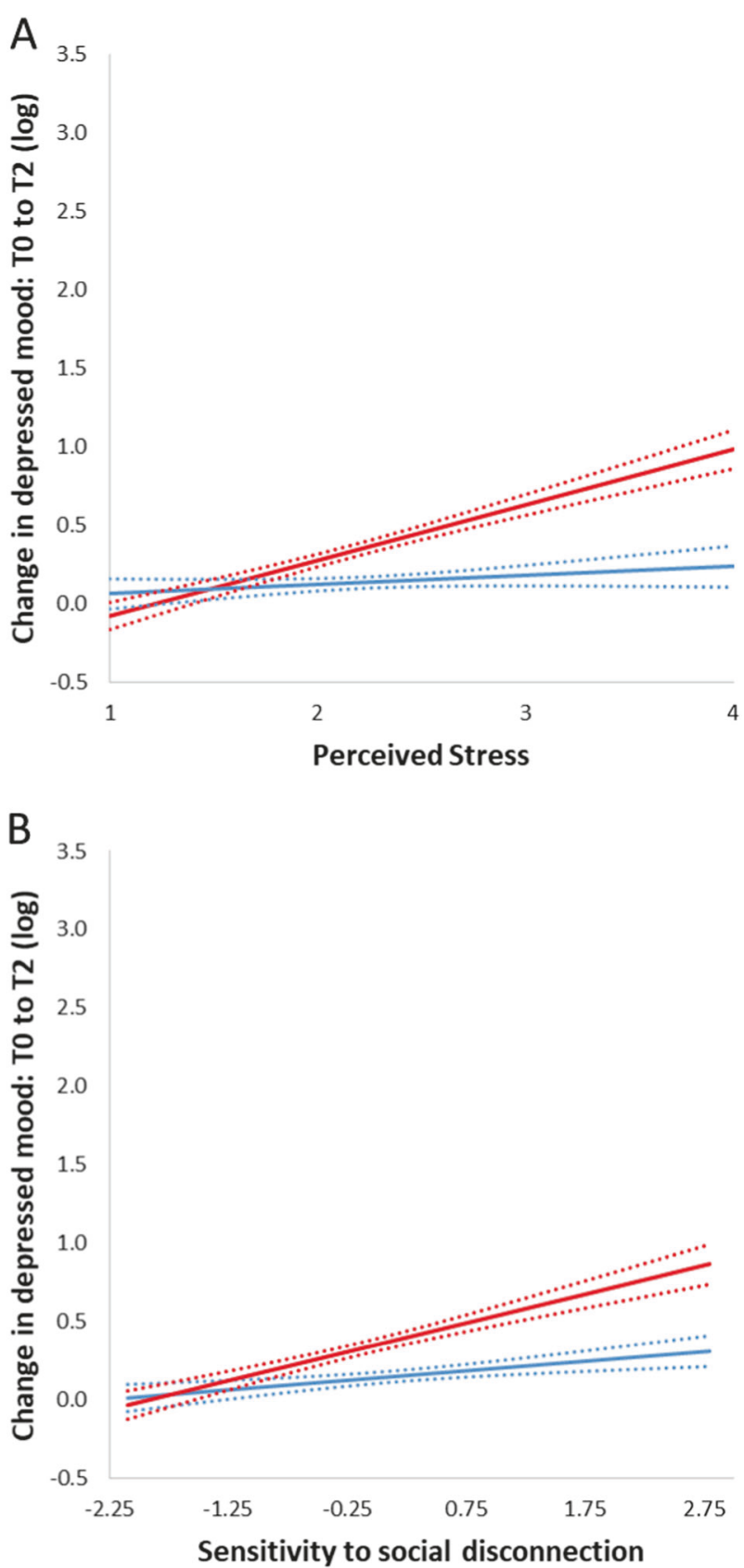

Fig. 1 Associations between change in depressed mood from T0 (baseline) to T2 and baseline levels of $\mathbf{A}$ perceived stress and $\mathbf{B}$ trait sensitivity to social disconnection in endotoxin (red) vs. placebo (blue) conditions, as illustrated by estimated regression lines by condition. Curvilinear brackets indicate residual standard error. For changes in depressed mood, results are presented as natural log transformed

activation-related transcription factors, EGR1 and EGR2. No gene transcripts were downregulated $>1.2$-fold in response to endotoxin. The magnitude of increase in TNF RNA concentrations from baseline to T0.5 correlated positively with the magnitude of increase in TNF protein concentrations from baseline to multiple follow-up time points, including T3 $(r=0.42, P<.001)$ and T6 $(r=$ $0.52, P<.001)$. TELiS bioinformatics was applied to all genes showing $>1.2$-fold difference in the magnitude of the interaction term for the selected socio-behavioral variables (For each moderator, specific genes are listed; Supplement, Differentially Expressed Genes) 

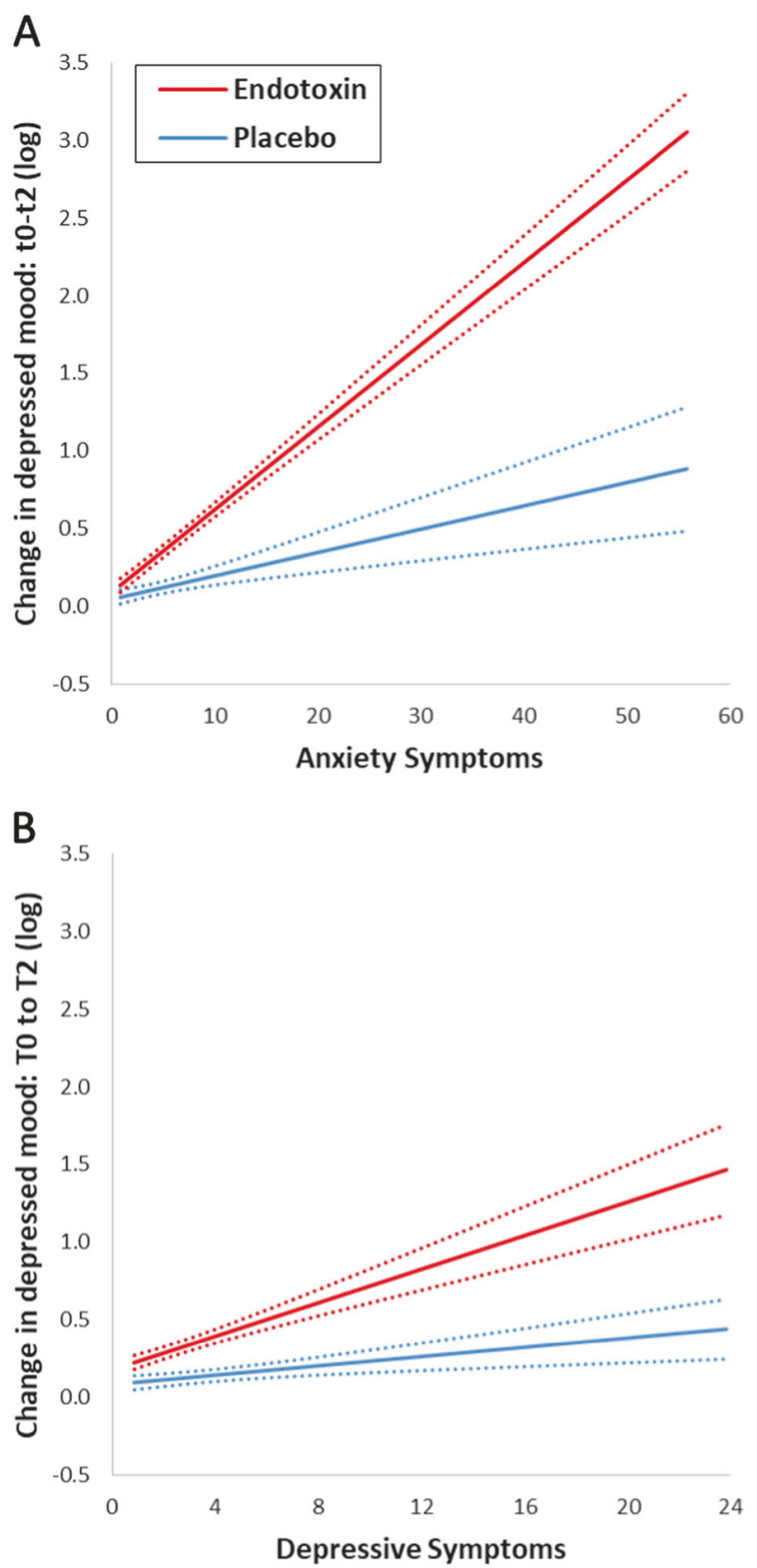

Fig. 2 Associations between change in depressed mood from T0 (baseline) to T2 and baseline levels of $\mathbf{A}$ anxiety symptoms and $\mathbf{B}$ depressive symptoms in endotoxin (red) vs. placebo (blue) conditions, as illustrated by estimated regression lines by condition. Curvilinear brackets indicate residual standard error. For changes in depressed mood, results are presented as natural log transformed

For socio-emotional variables, results indicated that perceived stress and sensitivity to social disconnection interacted with endotoxin, such that high levels of perceived stress predicted increased endotoxin-induced activation of both AP-1 $(P<0.01)$ and NF-KB $(P<0.01)$. As reported previously $[19,20]$, sensitivity to social disconnection also predicted increased activation of AP-1 $(P<0.02)$ and NF-KB $(P<0.02)$. (Fig. 3)

For the behavioral variables, depressive symptoms at baseline interacted with endotoxin such that higher levels of depressive symptoms predicted greater endotoxin-induced activation of AP-1
$(P<0.01)$ but not NF-KB $(P=0.09)$ (Fig. 3). However, for anxiety, no difference in endotoxin-induced activation of AP-1 $(P=0.06)$ or NF-KB $(P=0.59)$ was found.

Ancillary analyses found no significant interactions for any of the moderators on Type I IFN responses $(P>0.50)$.

\section{DISCUSSION}

In healthy adults, inflammatory exposure using endotoxin administration induced increases in depressed mood as compared to placebo. Consistent with the social signal transduction hypothesis of depression that links experiences of heightened social-environmental stress with increased inflammation to drive depression, the magnitude of the depression response was shaped by multiple socio-emotional factors and behavioral symptoms. Indeed, higher levels of perceived stress and sensitivity to social disconnection, along with baseline symptoms of anxiety and depression, were all found to moderate severity of depressed mood in response to endotoxin as compared to placebo, suggesting that perceptions of stress, sensitivity to social disconnection, and related anxiety and depressive symptoms lead to exaggerated affective vulnerability following inflammatory exposure. Importantly, among these factors, anxiety emerged as having a particularly salient role; anxiety symptoms prior to endotoxin exposure uniquely and strongly predicted those most at risk for increases in depressed mood, when taking all other factors into account.

Molecular analyses examined parallel variations in endotoxininduced activation of pro-inflammatory transcription control pathways (NF-KB and AP-1) in circulating immune cells. Results suggest that the moderating effects of baseline levels of perceived stress, sensitivity to social disconnection, and depressive symptoms on depressed mood, but not anxiety symptoms, may stem, at least in part, from increased leukocyte transcriptional response to endotoxin.

Prospective data have found that anxiety, sub-syndromal depressive symptoms, and sleep disturbance serve as risk factors or prodromal symptoms to identify those adults most likely to develop a depression [2, 3, 5, 26, 27]. Furthermore, anxiety and major depressive disorder show a high level of shared common variant risk [28]. This experimental study confirms the salience of anxiety and depressive symptoms for affective vulnerability and provides novel insight that these behavioral symptoms may increase depressed mood in response to inflammatory stimuli, in part by augmenting leukocyte inflammatory responses (i.e., depressive symptoms) or, in the absence of increases in leukocyte inflammatory responses, by augmenting affective sensitivity to inflammatory cytokines (i.e., anxiety symptoms). Previously, we found that the moderating role of sleep disturbance on depressed mood was limited to females [24]. However, severity of sleep disturbance in this sample of males and females may be too minimal to detect an effect.

Given that perceived stress, sensitivity to social disconnection, and depressive symptoms appear to regulate the magnitude of inflammatory response in part by increasing activity of proinflammatory transcription factors, targeting these specific sociobehavioral profiles by using precision-based therapeutic approaches and/or peripheral anti-inflammatory interventions may mitigate the risk for depression following exposure to a heightened inflammatory state (i.e., infectious challenge, psychological stress).

Several limitations require consideration. First, it is not known whether these findings generalize to older adults or to subgroups who are at elevated risk for depression (e.g., patients with anxiety disorders). Second, whereas endotoxin exposure can be used as an experimental probe to understand the role of inflammation in 
$\log _{2}$ TFBM ratio for Moderator $x$ Endotoxin Interaction

(endotoxin effect for high moderator value vs. low)

\begin{tabular}{|c|c|c|c|c|c|c|c|c|c|}
\hline Moderator & & -2 & -1.5 & -1 & -0.5 & 0 & 0.5 & 1 & 1.5 \\
\hline Perceived Stress & $\begin{array}{r}\text { NF-kB } \\
\text { AP-1 }\end{array}$ & 노사 & 1 & & & & $1 x$ & & 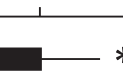 \\
\hline Social Disconnection & $\begin{array}{r}\text { NF-kB } \\
\text { AP-1 }\end{array}$ & & & & & & & & * \\
\hline Anxiety Symptoms & $\begin{array}{r}\text { NF-kB } \\
\text { AP-1 }\end{array}$ & & & & & & & & \\
\hline Depressive Symptoms & $\begin{array}{r}\text { NF-kB } \\
\text { AP-1 }\end{array}$ & & & & & & & & $* \mathrm{p}<.05$ \\
\hline
\end{tabular}

Fig. 3 Transcription factor-binding motif (TFBM) ratio for transcription factors, activator protein (AP)-1 and nuclear factor (NF)- $\mathrm{kB}$, as measured by TELiS promoter-based bioinformatic analyses of genes, in endotoxin vs. placebo condition. Selected covariates or moderators were those found to moderate changes in depressed mood following endotoxin exposure

sickness behaviors $[15,55]$, the main effect of endotoxin on depressed mood remains significant after controlling for sickness symptoms at T2 $[19,20]$. Third, future research is needed to evaluate whether differences in the relative prevalence of monocytes contribute to differences in inflammatory reactivity. Fourth, this study was not powered to resolve the quantitative strength of the moderators within the endotoxin group itself. Finally, acute increases in depressed mood in this experimental model do not indicate induction of clinical depression.

In summary, administration of endotoxin serves as an experimental approach to probe mood changes invoked by systemic inflammation, as well as the biological mechanisms of these effects. Given the acute and time-limited effects of endotoxin administration, implications for inflammatory biotype of depression, or for inflammatory conditions co-morbid with depression, are not yet fully appreciated. Nevertheless, the fact that multiple socio-behavioral factors which are independently associated with depression risk were also found to moderate both endotoxininduced depressive responses and gene transcriptional profiles underscores the value of this experimental model of the inflammatory biotype of depression. Further research using this experimental model will inform the mechanisms linking socioemotional and behavioral profiles to affective vulnerability and will provide new insights for development of varying and targeted therapeutic approaches to prevent or treat the inflammatory biotype of depression.

\section{ACKNOWLEDGEMENTS}

We thank Anthony Suffredini, MD, at the National Institutes of Health, Warren Grant Magnuson Clinical Center, for providing the standard reference endotoxin, the staff of the UCLA Clinical and Translational Research Center for providing technical assistance, the staff of the UCLA Cousins Center Inflammatory Biology Laboratory for laboratory and assay support, and the staff of the UCLA Statistical Consulting Group for providing statistical advice.

\section{ADDITIONAL INFORMATION}

Supplementary Information accompanies this paper at (https://doi.org/10.1038/ s41386-018-0259-6).

Funding and disclosure: This research was funded by an R01 from the National Institute of Mental Health to NIE (5R01MH091352). The authors also acknowledge the additional support provided by R01AG051944, R01AG026364, R01CA160245, and R01CA207130 to MRI; P30AG017265 and R01AG043404 to SWC; National Center for Advancing Translational Sciences UCLA CTSI Grant UL1TR001881; and the Cousins
Center for Psychoneuroimmunology. Additionally, HJC was supported by K23AG049085, NIH/NCATS UCLA CTSI Grant KL2TR000122, and NARSAD Young Investigator Grant from the Brain and Behavior Research Foundation. MRI, SC, HJC, and NIE have received funding from $\mathrm{NIH}$. The other authors declare no competing interests.

Publisher's note: Springer Nature remains neutral with regard to jurisdictional claims in published maps and institutional affiliations.

\section{REFERENCES}

1. Kessler RC, Berglund $P$, Demler $O$, Jin R, Merikangas KR, Walters EE. Lifetime prevalence and age-of-onset distributions of DSM-IV disorders in the national comorbidity survey replication. Arch Gen Psychiatry. 2005;62:593-602.

2. Slavich GM, Irwin MR. From stress to inflammation and major depressive disorder: a social signal transduction theory of depression. Psychol Bull. 2014;140: 774-815.

3. Cho HJ, Lavretsky H, Olmstead R, Levin MJ, Oxman MN, Irwin MR. Sleep disturbance and depression recurrence in community-dwelling older adults: a prospective study. Am J Psychiatry. 2008;165:1543-50.

4. Irwin MR. Why sleep is important for health: a psychoneuroimmunology perspective. Annu Rev Psychol. 2015;66:143-72.

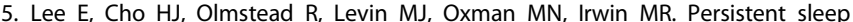
disturbance: a risk factor for recurrent depression in community-dwelling older adults. Sleep. 2013;36:1685-91.

6. Irwin MR, Miller AH. Depressive disorders and immunity: 20 years of progress and discovery. Brain Behav Immun. 2007;21:374-83.

7. Dowlati Y, Herrmann N, Swardfager W, Liu H, Sham L, Reim EK, et al. A metaanalysis of cytokines in major depression. Biol Psychiatry. 2010;67:446-57.

8. Padmos RC, Hillegers MH, Knijff EM, Vonk R, Bouvy A, Staal FJ, et al. A discriminating messenger RNA signature for bipolar disorder formed by an aberrant expression of inflammatory genes in monocytes. Arch Gen Psychiatry. 2008;65:395-407.

9. Savitz J, Frank MB, Victor T, Bebak M, Marino JH, Bellgowan PS, et al. Inflammation and neurological disease-related genes are differentially expressed in depressed patients with mood disorders and correlate with morphometric and functional imaging abnormalities. Brain Behav Immun. 2013;31:161-71.

10. Raison $C L$, Rutherford RE, Woolwine BJ, Shuo C, Schettler P, Drake DF, et al. A randomized controlled trial of the tumor necrosis factor antagonist infliximab for treatment-resistant depression: the role of baseline inflammatory biomarkers. JAMA Psychiatry. 2013;70:31-41.

11. Eller T, Vasar V, Shlik J, Maron E. Pro-inflammatory cytokines and treatment response to escitalopram in major depressive disorder. Prog Neuropsychopharmacol Biol Psychiatry. 2008;32:445-50.

12. Uher R, Tansey KE, Dew T, Maier W, Mors $\mathrm{O}$, Hauser J, et al. An inflammatory biomarker as a differential predictor of outcome of depression treatment with escitalopram and nortriptyline. Am J Psychiatry. 2014;171:1278-86.

13. Kruse JL, Congdon E, Olmstead R, Njau S, Breen EC, Narr KL, et al. Inflammation and improvement of depression following electroconvulsive therapy in treatment resistant depression. J Clin Psychiatry. 2018;79:17-23. 
14. Gimeno D, Kivimaki M, Brunner EJ, Elovainio $M$, De Vogli R, Steptoe $A$, et al. Associations of c-reactive protein and interleukin- 6 with cognitive symptoms of depression: 12-year follow-up of the Whitehall II study. Psychol Med. 2009;39:413-23.

15. DellaGioia N, Hannestad J. A critical review of human endotoxin administration as an experimental paradigm of depression. Neurosci Biobehav Rev. 2010;34: 130-43.

16. Eisenberger $\mathrm{NI}$, Berkman ET, Inagaki TK, Rameson LT, Mashal NM, Irwin MR. Inflammation-induced anhedonia: endotoxin reduces ventral striatum responses to reward. Biol Psychiatry. 2010;68:748-54.

17. Eisenberger NI, Inagaki TK, Rameson LT, Mashal NM, Irwin MR. An fMRI study of cytokine-induced depressed mood and social pain: the role of sex differences. Neuroimage. 2009;47:881-90.

18. Kullmann JS, Grigoleit JS, Wolf OT, Engler H, Oberbeck R, Elsenbruch S, et al Experimental human endotoxemia enhances brain activity during social cognition. Soc Cogn Affect Neurosci. 2014;9:786-93.

19. Moieni M, Irwin MR, Jevtic I, Breen EC, Cho HJ, Arevalo JM, et al. Trait sensitivity to social disconnection enhances pro-inflammatory responses to a randomized controlled trial of endotoxin. Psychoneuroendocrinology. 2015;62:336-42.

20. Moieni M, Irwin MR, Jevtic I, Olmstead R, Breen EC, Eisenberger NI. Sex differences in depressive and socioemotional responses to an inflammatory challenge: implications for sex differences in depression. Neuropsychopharmacology. 2015;40:1709-16.

21. Lotrich FE, Ferrell RE, Rabinovitz M, Pollock BG. Risk for depression during interferon-alpha treatment is affected by the serotonin transporter polymorphism. Biol Psychiatry. 2009;65:344-8.

22. Bull SJ, Huezo-Diaz P, Binder EB, Cubells JF, Ranjith G, Maddock C, et al. Functional polymorphisms in the interleukin- 6 and serotonin transporter genes, and depression and fatigue induced by interferon-alpha and ribavirin treatment. Mol Psychiatry. 2009;14:1095-104.

23. Harrison NA, Brydon L, Walker C, Gray MA, Steptoe A, Critchley HD. Inflammation causes mood changes through alterations in subgenual cingulate activity and mesolimbic connectivity. Biol Psychiatry. 2009;66:407-14.

24. Cho HJ, Eisenberger NI, Olmstead R, Breen EC, Irwin MR. Preexisting mild sleep disturbance as a vulnerability factor for inflammation-induced depressed mood: a human experimental study. Transl Psychiatry. 2016;6:e750.

25. O'Connor M-F, Bower JE, Cho HJ, Creswell JD, Dimitrov S, Hamby ME, et al. To assess, to control, to exclude: effects of biobehavioral factors on circulating inflammatory markers. Brain Behav Immun. 2009;23:887-97.

26. Beesdo K, Bittner A, Pine DS, Stein MB, Hofler M, Lieb R, et al. Incidence of social anxiety disorder and the consistent risk for secondary depression in the first three decades of life. Arch Gen Psychiatry. 2007;64:903-12.

27. Stein MB, Fuetsch M, Muller N, Hofler M, Lieb R, Wittchen HU. Social anxiety disorder and the risk of depression: a prospective community study of adolescents and young adults. Arch Gen Psychiatry. 2001;58:251-6.

28. Brainstorm C, Anttila V, Bulik-Sullivan B, Finucane HK, Walters RK, Bras J, et al. Analysis of shared heritability in common disorders of the brain. Science. 2018. https://doi.org/10.1126/science.aap8757.

29. Irwin MR, Cole SW. Reciprocal regulation of the neural and innate immune systems. Nat Rev Immunol. 2011;11:625-32.

30. Cole SW, Arevalo JM, Takahashi R, Sloan EK, Lutgendorf SK, Sood AK, et al. Computational identification of gene-social environment interaction at the human il6 locus. Proc Natl Acad Sci USA. 2010;107:5681-6.

31. Slavich GM, Cole SW. The emerging field of human social genomics. Clin Psychol Sci. 2013;1:331-48.

32. Muscatell KA, Moieni M, Inagaki TK, Dutcher JM, Jevtic I, Breen EC, et al. Exposure to an inflammatory challenge enhances neural sensitivity to negative and positive social feedback. Brain Behav Immun. 2016;51:21-29.
33. Inagaki TK, Muscatell KA, Irwin MR, Moieni M, Dutcher JM, Jevtic I, et al. The role of the ventral striatum in inflammatory-induced approach toward support figures. Brain Behav Immun. 2015;44:247-52.

34. Suffredini AF, Hochstein HD, McMahon FG. Dose-related inflammatory effects of intravenous endotoxin in humans: evaluation of a new clinical lot of Escherichia coli o-113 endotoxin. J Infect Dis. 1999;179:1278-82.

35. Grigoleit JS, Kullmann JS, Wolf OT, Hammes F, Wegner A, Jablonowski S, et al. Dose-dependent effects of endotoxin on neurobehavioral functions in humans. PLoS ONE. 2011;6:e28330.

36. Ishihara K, Hirano T. II-6 in autoimmune disease and chronic inflammatory proliferative disease. Cytokine Growth Factor Rev. 2002;13:357-68.

37. Steptoe A, Hamer M, Chida Y. The effects of acute psychological stress on circulating inflammatory factors in humans: a review and meta-analysis. Brain Behav Immun. 2007;21:901-12.

38. Breen EC, Rezai AR, Nakajima K, Beall GN, Mitsuyasu RT, Hirano T, et al. Infection with HIV is associated with elevated IL-6 levels and production. J Immunol. 1990;144:480-4.

39. Baker F, Denniston M, Zabora J, Polland A, Dudley WN. A POMS short form for cancer patients: psychometric and structural evaluation. Psychooncology. 2002;11:273-81.

40. Cohen S, Kamarck T, Mermelstein R. A global measure of perceived stress. J Health Soc Behav. 1983;24:385-96.

41. Repetti RL, Taylor SE, Seeman TE. Risky families: family social environments and the mental and physical health of offspring. Psychol Bull. 2002;128:330-66.

42. Operario D, Adler NE, Williams DR. Subjective social status: reliability and predictive utility for global health. Psychol Health. 2004;19:237-46.

43. Sarason IG, Levine HM, Basham RB. Assessing social support: the social support questionnaire. J Pers Soc Psychol. 1983;44:127-39.

44. Loo R. A psychometric investigation of the Eysenck personality questionnaire. J Personal Assess. 1979;43:54-58.

45. Duke D, Krishnan M, Faith M, Storch EA. The psychometric properties of the brief fear of negative evaluation scale. J Anxiety Disord. 2006;20:807-17.

46. Mehrabian A. Evidence bearing on the affiliative tendency (MAFF) and sensitivity to rejection (MSR) scales. Curr Psychol. 1994;13:97-16.

47. Russell D, Peplau LA, Cutrona CE. The revised UCLA loneliness scale: concurrent and discriminant validity evidence. J Pers Soc Psychol. 1980;39:472-80.

48. Fraley RC, Waller NG, Brennan KA. An item response theory analysis of self-report measures of adult attachment. J Pers Soc Psychol. 2000;78:350-65.

49. Beck AT, Steer RA, Ball R, Ranieri W. Comparison of Beck depression inventories-IA and -II in psychiatric outpatients. J Personal Assess. 1996;67:588-97.

50. Buysse DJ, Reynolds CF 3rd, Monk TH, Berman SR, Kupfer DJ. The Pittsburgh Sleep Quality Index: a new instrument for psychiatric practice and research Psychiatry Res. 1989;28:193-13.

51. Bolstad BM, Irizarry RA, Astrand M, Speed TP. A comparison of normalization methods for high density oligonucleotide array data based on variance and bias. Bioinformatics. 2003;19:185-93.

52. Cole SW, Yan W, Galic Z, Arevalo J, Zack JA. Expression-based monitoring of transcription factor activity: the TELiS database. Bioinformatics. 2005;21: 803-10.

53. Miller GE, Chen E, Fok AK, Walker H, Lim A, Nicholls EF, et al. Low early-life social class leaves a biological residue manifested by decreased glucocorticoid and increased proinflammatory signaling. Proc Natl Acad Sci USA. 2009;106: 14716-21.

54. Efron B, Tibshirani RJ. An introduction to the bootstrap. New York, NY: Chapman and Hall; 1993.

55. Schedlowski M, Engler H, Grigoleit JS. Endotoxin-induced experimental systemic inflammation in humans: a model to disentangle immune-to-brain communication. Brain Behav Immun. 2014;35:1-8. 